\title{
Biexciton Emission from Edges and Grain Boundaries of Triangular $\mathrm{WS}_{2}$ Monolayers
}

Min Su Kim ${ }^{\dagger}$, Seok Joon Yun ${ }^{\dagger, \#}$, Yongjun Lee ${ }^{\dagger, \#}$, Changwon Seo ${ }^{\dagger, \#}$, Gang Hee Han ${ }^{\dagger}$, Ki Kang $\operatorname{Kim}^{\dagger, \perp}$, Young Hee Lee ${ }^{\dagger, \#}$, Jeongyong Kim ${ }^{*, \dagger, \#}$

${ }^{\dagger}$ Center for Integrated Nanostructure Physics (CINAP), Institute for Basic Science (IBS), Sungkyunkwan University, Suwon 440-746, Republic of Korea

\#Department of Energy Science, Sungkyunkwan University, Suwon 440-746, Republic of Korea

${ }^{\perp}$ Department of Energy and Materials Engineering, Dongguk University, Seoul 100-715,

Republic of Korea

*Email: j.kim@skku.edu 
Confocal Raman spectral mapping was used to identify the number of $\mathrm{WS}_{2}$ layers and confirm their uniformity. ${ }^{1-3}$ Consistent peak positions and a shift in the in-plane $\left(E_{2 g}^{1}\right)$ and out-of-plane $\left(\mathrm{A}_{1 \mathrm{~g}}\right)$ Raman modes of the vibrations in each $\mathrm{WS}_{2}$ sample were observed. ${ }^{2,3}$ The wavenumber difference between the $\mathrm{E}_{2 \mathrm{~g}}^{1}$ and $\mathrm{A}_{1 \mathrm{~g}}$ peaks in the Raman spectrum has been widely used to identify the number of $\mathrm{WS}_{2}$ crystalline layers because the value of this difference varies as a function of the number of layers. The frequency difference between these two peaks for $\mathrm{WS}_{2}$ was observed to be approximately $61 \mathrm{~cm}^{-1}$, i.e., the difference between $356 \mathrm{~cm}^{-1}$ for the $\mathrm{E}_{2 \mathrm{~g}}^{1}$ mode and $416.7 \mathrm{~cm}^{-1}$ for the $\mathrm{A}_{1 \mathrm{~g}}$ mode, which is consistent with a $\mathrm{WS}_{2}$ monolayer. $^{2}$ Figure $\mathrm{S} 1$ a shows the Raman intensity ratio $\left(I_{\mathrm{E} 12 \mathrm{~g}} / I_{\mathrm{A} 1 \mathrm{~g}}\right.$ peak $)$ map of the $\mathrm{WS}_{2}$ monolayer, and implies good thickness uniformity. Figure S1b shows the average Raman spectra of the edge and inner regions of the $\mathrm{WS}_{2}$ monolayer. Both Raman intensities were found to be almost the same, indicative of good thickness uniformity.
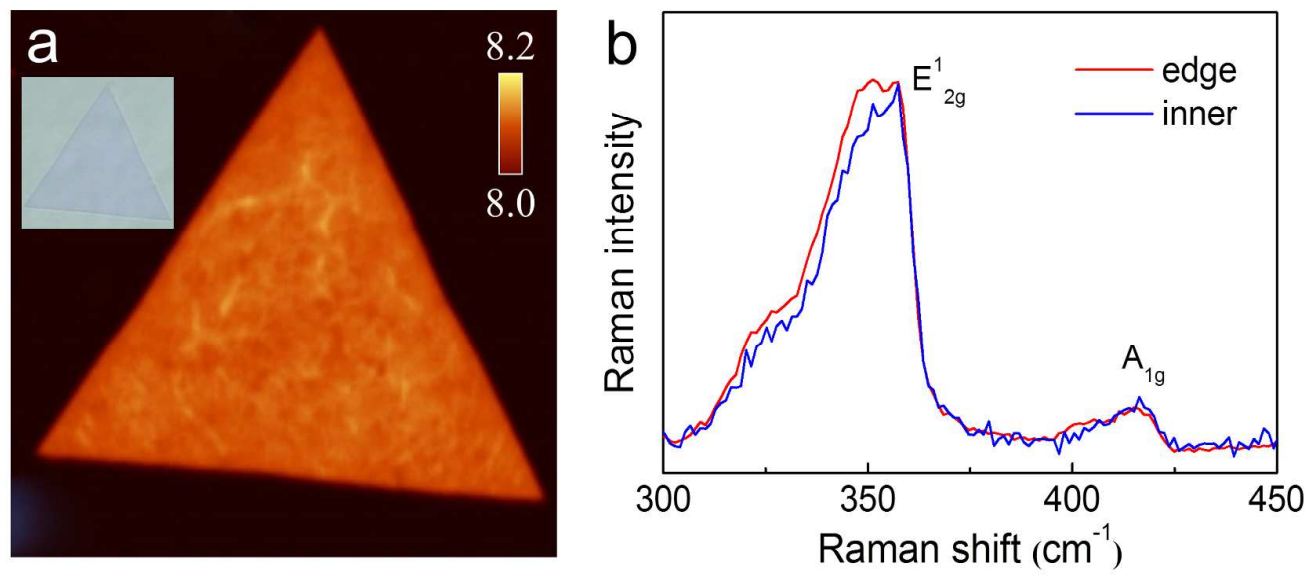

Figure S1. Confirmation of the number of layers in the $\mathrm{WS}_{2}$ monolayer: (a) Raman intensity ratio $\left(\mathrm{I}_{\mathrm{E} 12 \mathrm{~g}} / \mathrm{I}_{\mathrm{A} 1 \mathrm{~g}}\right)$ map. The inset shows an optical microscope image. (b) Raman spectra of the edge and the inner regions of the $\mathrm{WS}_{2}$ monolayer. 

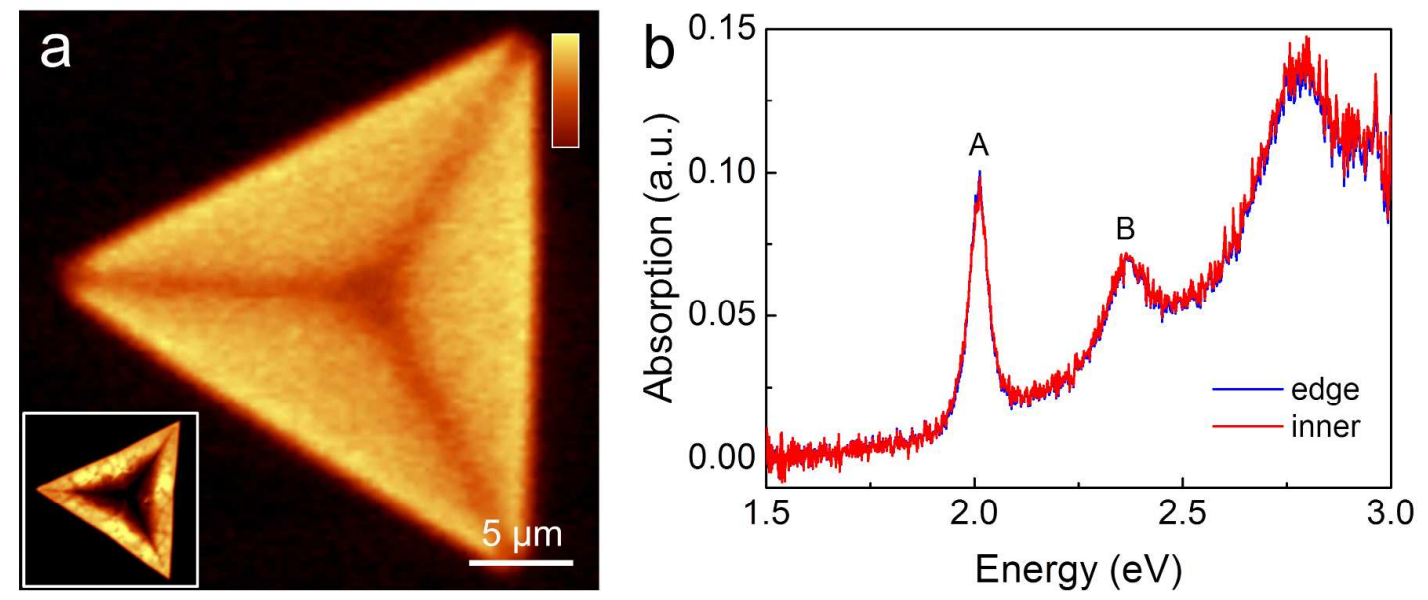

Figure S2. (a) Absorption intensity map of triangular $\mathrm{WS}_{2}$ monolayer. The inset shows $\mathrm{PL}$ intensity map of the same triangular $\mathrm{WS}_{2}$ monolayer. Somewhat less (only by $\sim 30 \%$ ) absorption was measured along the lines from the vertices to the center, where the initial growth is known to occur in triangular TMD monolayers. Except on these lines, absorption is uniform over the triangular $\mathrm{WS}_{2}$ grain. (b) Representative absorption spectra obtained from the edge region and the inner region of the triangular $\mathrm{WS}_{2}$ monolayer. 

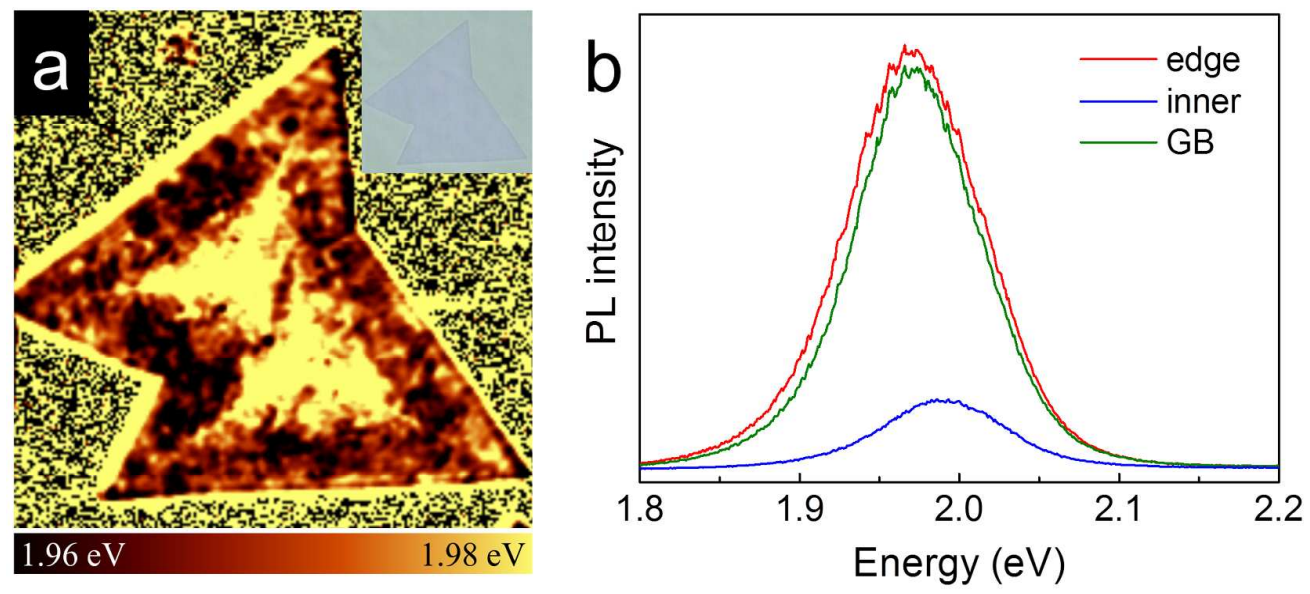

Figure S3. PL spectrum variation in a polycrystalline $\mathrm{WS}_{2}$ monolayer. (a) A PL peak position map. The inset shows an optical microscope image. (b) Representative PL spectra obtained from the edge region, the inner region, and the GB. 

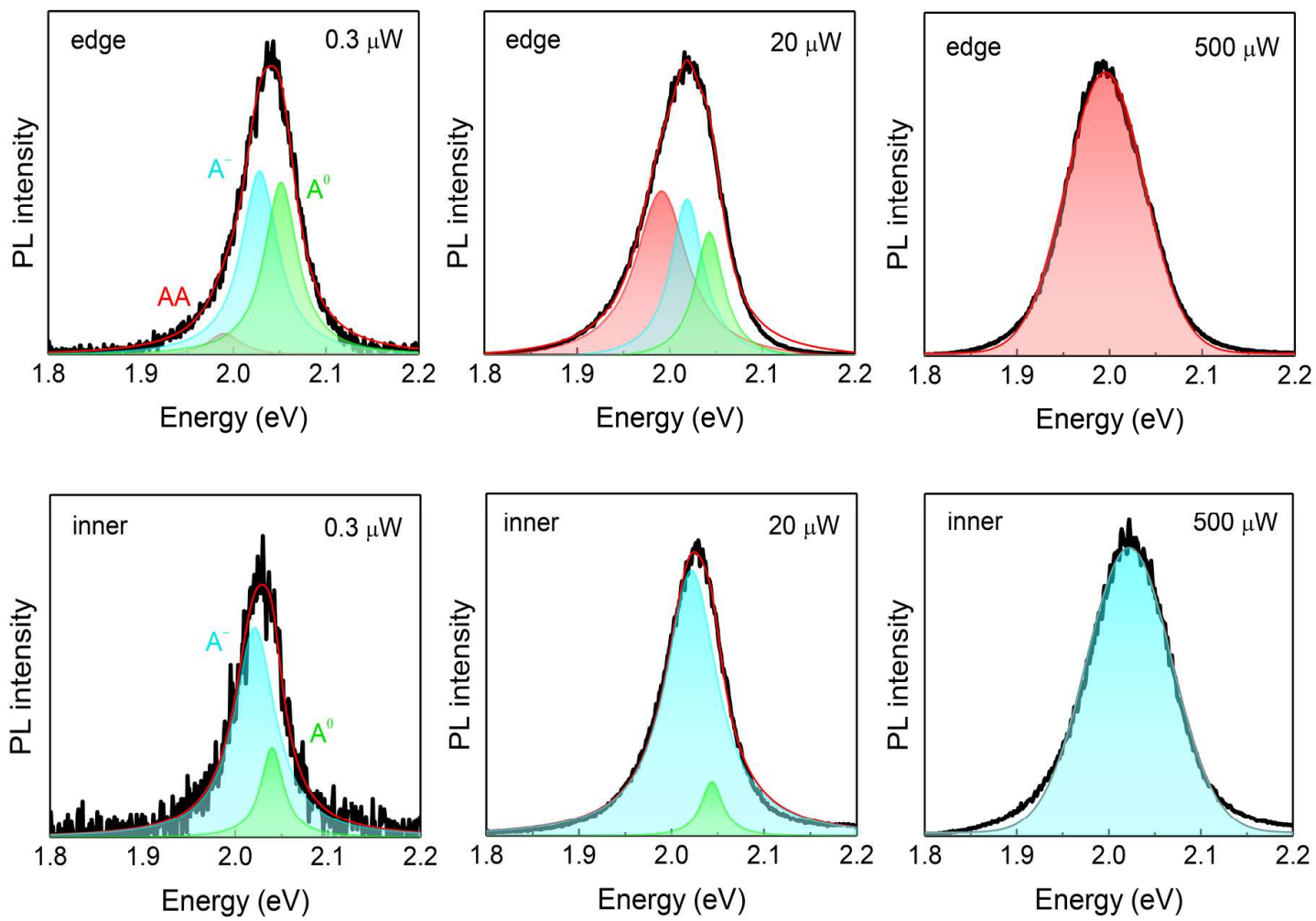

Figure S4. Deconvoluted spectra obtained from the inner region and edge region for each of three laser power values obtained by fitting with peaks of neutral excitons $\left(\mathrm{A}^{0}\right)$, trions $\left(\mathrm{A}^{-}\right)$ and biexcitons (AA). 


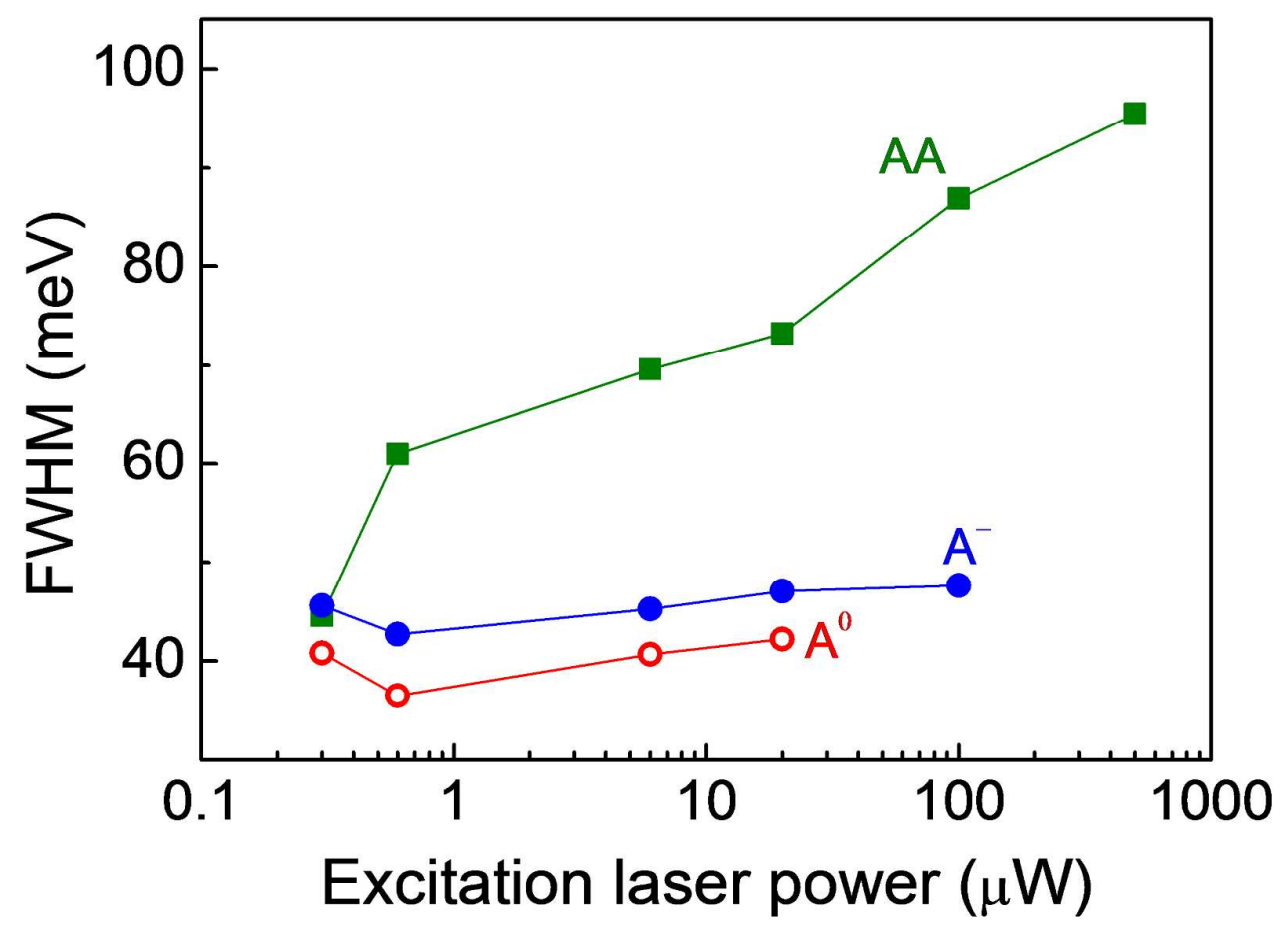

Figure S5. FWHM of deconvoluted PL peaks of neutral excitons $\left(\mathrm{A}^{0}\right)$, trions $\left(\mathrm{A}^{-}\right)$and biexcitons (AA) as a function of excitation laser power. The solid lines are guides to the eye. Note that FWHMs of biexciton peaks were about twice of neutral exciton peaks, except for the $0.3 \mu \mathrm{W}$ where the biexciton peak was negligibly weak. 

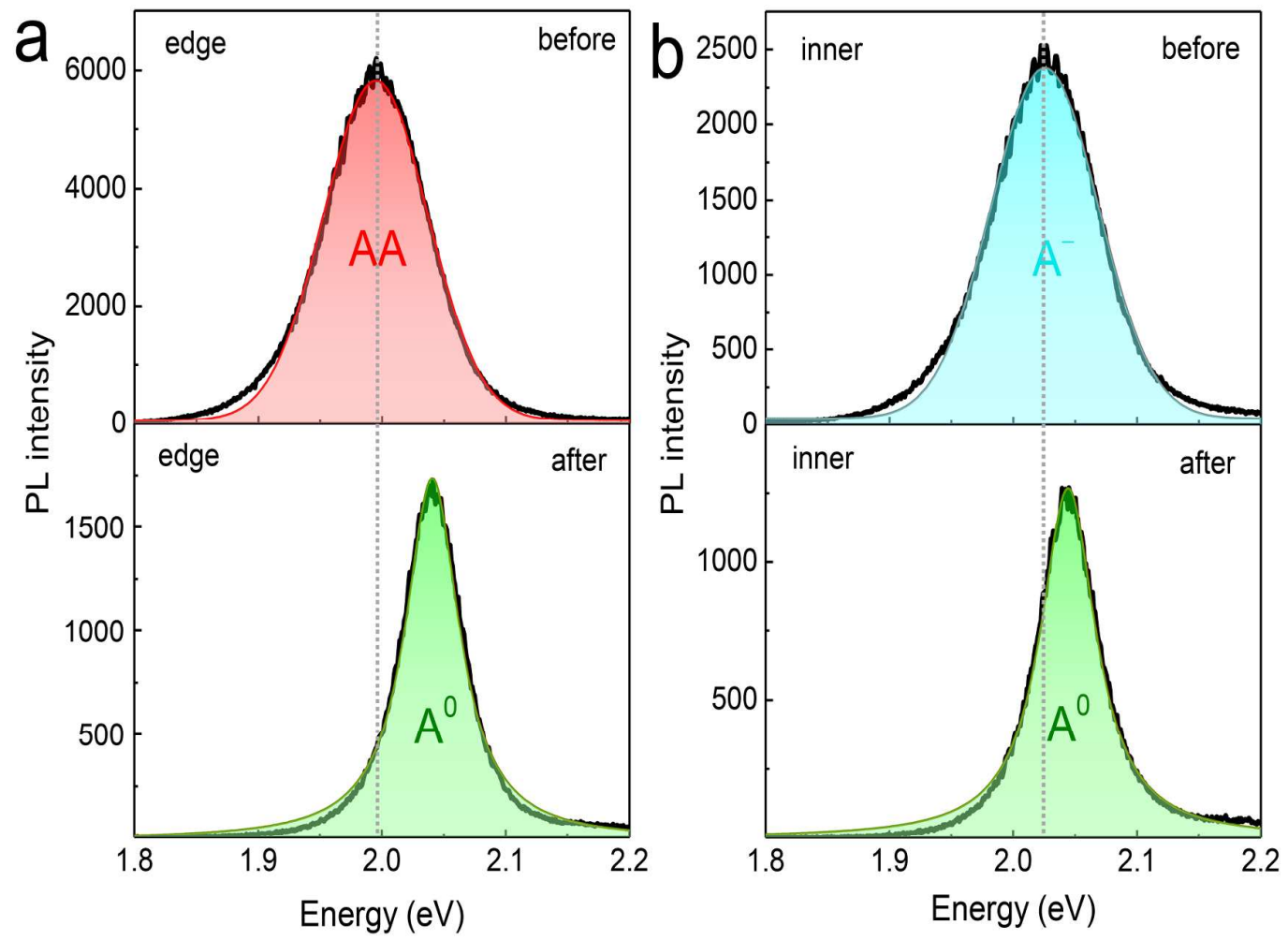

Figure S6. PL spectra of pristine (top figure) and heat-treated (bottom figure) $\mathrm{WS}_{2}$ monolayers obtained from (a) the edge region and (b) the inner region. 
The strong Raman peak of the $\mathrm{WS}_{2}$ monolayer consists of two modes, 2LA(M) and $\mathrm{E}_{2 \mathrm{~g} .}^{1}{ }^{2,3}$ The multi-peak Lorentzian fitting shown in Figure S5 clearly separated the 2LA(M) and $\mathrm{E}_{2 \mathrm{~g}}^{1}$ modes. The longitudinal acoustic phonons LA(M) are in-plane collective movements of the atoms in the lattice, similar to that of sound waves, which are periodic compressions and expansions of the lattice that occur along the direction of propagation. In addition, the full width at half maximum (FWHM) of the $E_{2 g}^{1}$ mode at the edge and inner regions decreased from 7.6 to $5.4 \mathrm{~cm}^{-1}$ and from 6.7 to $5.6 \mathrm{~cm}^{-1}$, respectively. These increases in the 2LA(M) mode intensity and $\mathrm{E}^{1}{ }_{2 \mathrm{~g}}$ mode FWHM imply the presence of lattice disorder due to an $\mathrm{S}$ vacancy. ${ }^{2,4}$
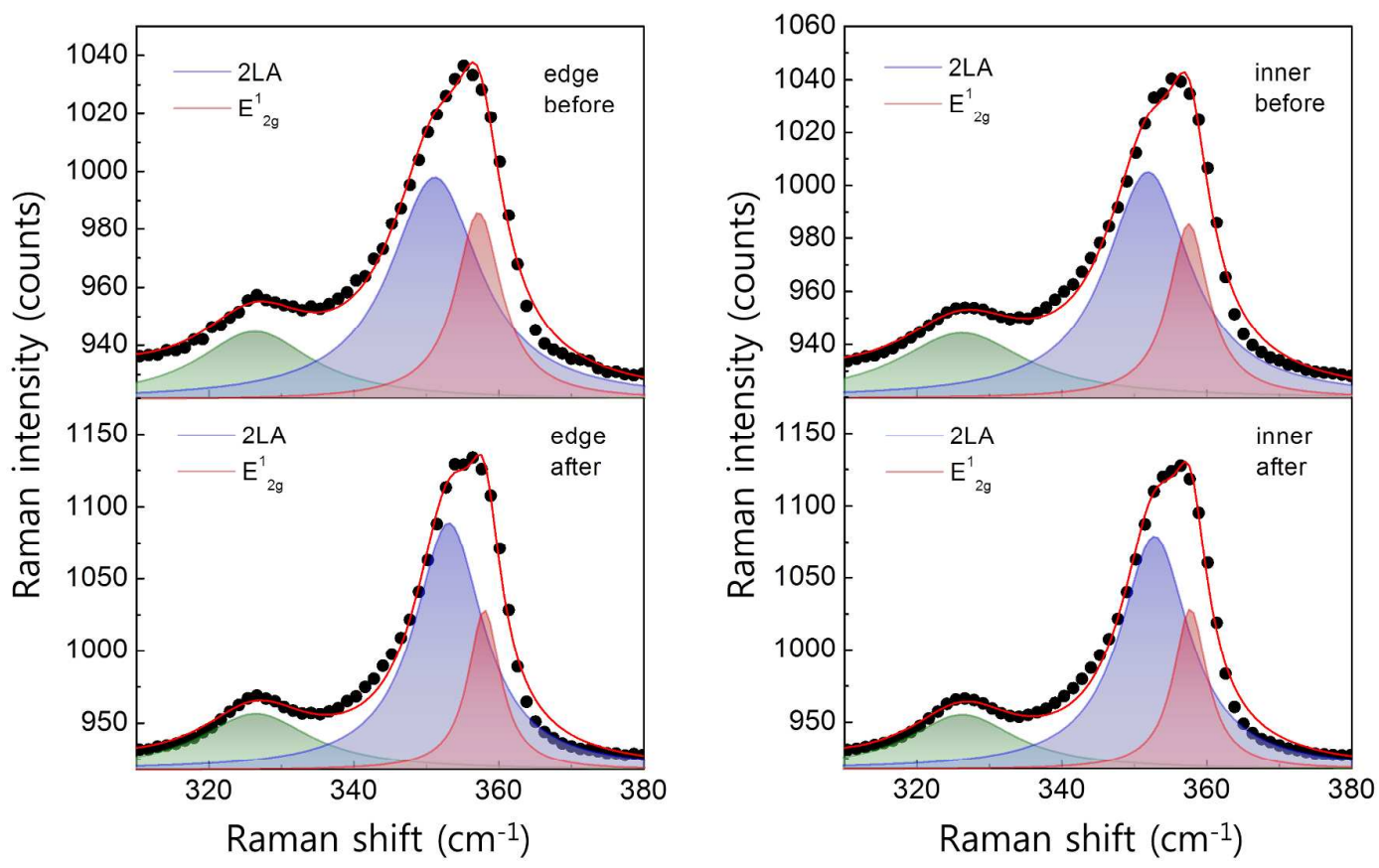

Figure S7. Multi-peak Lorentzian fittings of the Raman peaks of pristine and heat-treated $\mathrm{WS}_{2}$ monolayers obtained from (a) the edge region and (b) the inner region. 

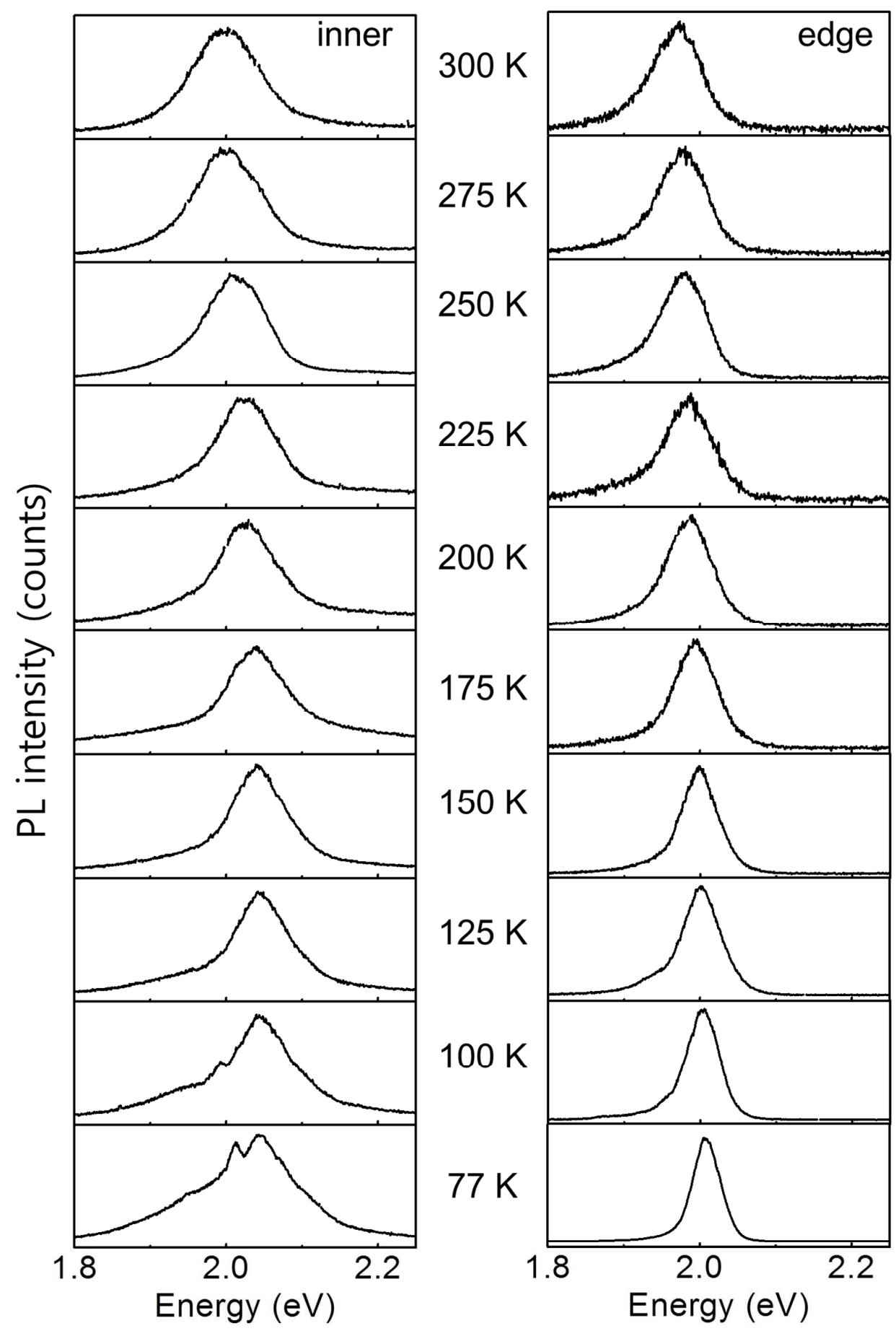

Figure S8. Temperature-dependent PL spectra obtained from the inner region and the edge region of the $\mathrm{WS}_{2}$ monolayer. 


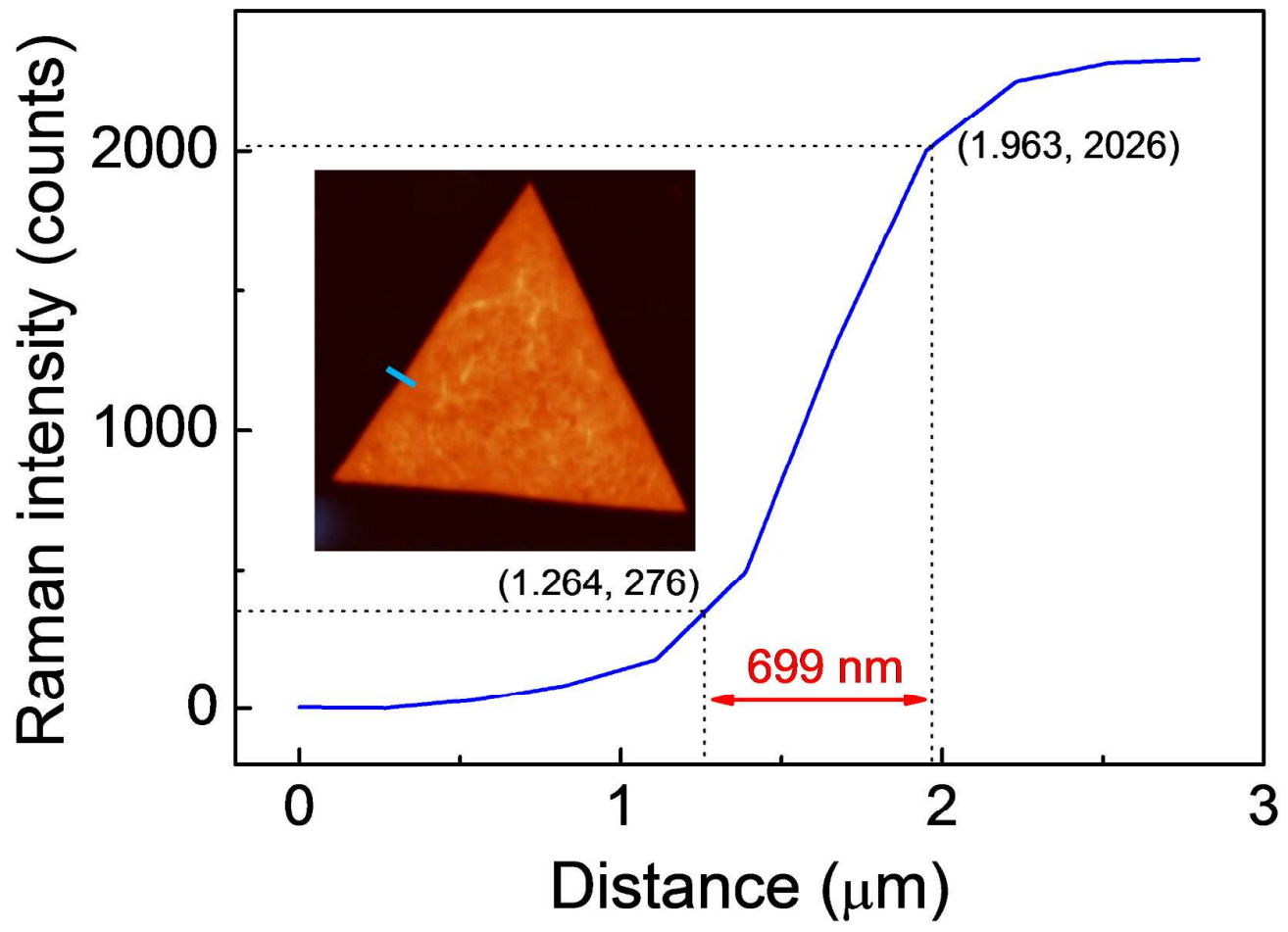

Figure S9. Raman intensity profile along the blue line in the inserted Raman intensity map of triangular $\mathrm{WS}_{2}$ monolayer. 


\section{Supplementary Information Reference}

1. Gutiérrez, H. R.; Perea-López, N.; Elías, A. L.; Berkdemir, A.; Wang, B.; Lv, R.; LópezUrías, F.; Crespi, V. H.; Terrones, H.; Terrones, M. Extraordinary Room-Temperature Photoluminescence in Triangular $\mathrm{WS}_{2}$ Monolayers. Nano Lett. 2013, 13, 3447-3454.

2. Berkdemir, A.; Gutiérrez, H. R.; Botello-Méndez, A. R.; Perea-López, N.; Elías, A. L.; Chia, C.-I.; Wang, B.; Crespi, V. H.; López-Urías, F.; Charlier, J.-C.; Terrones, H.; Terrones, M. Identification of Individual and Few Layers of $\mathrm{WS}_{2}$ Using Raman Spectroscopy. Sci. Rep. 2013, 3, 1755.

3. Zhao, W.; Ghorannevis, Z.; Amara, K. K.; Pang, J. R.; Toh, M.; Zhang, X.; Kloc, C.; Tan, P. H.; Eda, G. Lattice Dynamics in Mono- and Few-Layer Sheets of $\mathrm{WS}_{2}$ and $\mathrm{WSe}_{2}$. Nanoscale 2013, 5, 9677-9683.

4. Tongay, S.; Zhou, J.; Ztaca, C.; Liu, J.; Kang, J. S.; Matthews, T. S.; You, L.; Li, J.; Grossman, J. C.; Wu, J. Broad-Range Modulation of Light Emission in TwoDimensional Semiconductors by Molecular Physisorption Gating. Nano Lett. 2013, 13, 2831-2836. 